\title{
An Efficient Method for Generating Autocorrelated Non-Gaussian Sequences
}

\author{
José Cândido Silveira Santos Filho and Michel Daoud Yacoub
}

\begin{abstract}
We propose a novel method for generating random sequences matching both a target non-Gaussian distribution and a target autocorrelation. In the proposed scheme, samples matching the target distribution are drawn independently and then suitably rearranged to match the target autocorrelation. Our method overcomes the difficulties of the standard method in generating sequences with unknown inverse distribution. Besides communications, our results find applicability in many different areas concerned with non-Gaussian processes. Many examples are included.
\end{abstract}

Keywords-Autocorrelation, non-Gaussian processes, order statistics, rank statistics, simulation.

\section{INTRODUCTION}

Modern communication systems are increasingly complex, and their analysis, design, and optimization usually lay hold of computer simulations. Analytical tools are certainly preferable, but many times they either tackle the problem in a simplistic manner or else prove impracticable. In such cases, simulation may be the only appropriate solution.

Most communication systems involve random phenomena, which are usually specified by means of a cumulative distribution function (CDF) and an autocorrelation coefficient (ACC) ${ }^{1}$. As a result, a typical simulation task is the generation of a random sequence matching a target $\mathrm{CDF}$ and a target $\mathrm{ACC}$. If the target CDF is Gaussian, the solution is straightforward, because a linearly filtered Gaussian sequence remains Gaussian [1], [2]. Passing a white Gaussian sequence through an appropriate linear filter, one can achieve the target ACC while preserving the target Gaussian CDF. However, if the target $\mathrm{CDF}$ is non-Gaussian ${ }^{2}$, the solution becomes more intricate [6], [7]. Linear filtering no longer applies, because it does not preserve non-Gaussian CDFs. For instance, by linearly filtering a white exponential sequence, the colored output sequence is no longer exponential. And this holds true for any nonGaussian sequence. Therefore, other techniques are required in the non-Gaussian case.

The standard method for generating an autocorrelated nonGaussian sequence is to modify an autocorrelated Gaussian sequence by applying to it a memoryless nonlinear transformation [7]. The transformation is chosen so that the target CDF is attained, and the Gaussian ACC is chosen so that

The authors are with the Department of Communications, School of Electrical and Computer Engineering, University of Campinas, PO Box 6101, 13083-852 Campinas, SP, Brazil, Tel: +55 (19) 3788 5106, Fax: +55 (19) 3289 1395, E-mail:\{candido,michel\} @wisstek.org.

${ }^{1}$ Equivalently, an autocovariance function (ACF) can be specified. Indeed, the ACC is the variance-normalized ACF.

${ }^{2}$ Sample examples of non-Gaussian processes occurring in communications are given in [3]-[5]. Other examples are the common fading channel models, including Rayleigh, Rice, Hoyt, Nakagami, Weibull, and lognormal. the target ACC is attained. In this method, the knowledge of the inverse of the target CDF is paramount; it is required to establish both the appropriate nonlinear transformation and the appropriate Gaussian ACC. However, the inverse CDF is sometimes unavailable. The gamma and beta CDFs, for instance, which find large applicability in engineering, have no closed-form inverse [8]. In such cases, the CDF must be inverted numerically, a task that may be neither simple nor attractive [9].

In this work, we propose a novel method for generating autocorrelated non-Gaussian sequences. In the proposed scheme, samples matching the target CDF are drawn independently and then suitably rearranged to match the target ACC. The inverse of the target CDF may be used if available, but it is indeed dispensable. Therefore, our method is particularly attractive when the inverse CDF is unknown. Besides communications, our method finds applicability in many different areas concerned with non-Gaussian processes.

In Section II, the standard method is revisited. The novel method is presented and justified in Sections III and IV, respectively. Finally, examples are given in Section V.

\section{StAndard Method}

We examine the problem of generating an $L$-sample realization sequence $\left\{y_{n}\right\}$ for a discrete-time random process $\left\{Y_{n}\right\}$ having a target non-Gaussian $\mathrm{CDF} F_{Y}(\cdot)$ and a target ACC

$$
\rho_{Y}(m) \triangleq \frac{E\left[Y_{n} Y_{n+m}\right]-\mu^{2}}{\sigma^{2}}
$$

where $m$ is a discrete-time lag, $E[\cdot]$ denotes expectation, $\mu$ is the mean value of $Y_{n}$, and $\sigma^{2}$ is the variance. The standard solution is as follows [7]:

1) Generate an $L$-sample realization sequence $\left\{x_{n}\right\}$ for a zero-mean unit-variance Gaussian process $\left\{X_{n}\right\}$ with an appropriate ACC $\rho_{X}(\cdot)$.

2) Apply a memoryless nonlinear transformation to $\left\{x_{n}\right\}$, forming $\left\{y_{n}\right\}$.

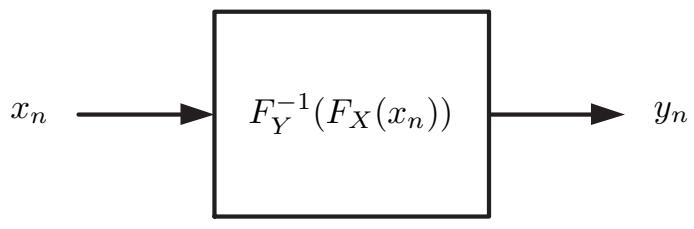

Fig. 1. The standard method for generating autocorrelated non-Gaussian sequences. 
The scheme is illustrated in Fig. 1. In order to attain the target $F_{Y}(\cdot)$, the nonlinearity is chosen to be [7]

$$
y_{n}=F_{Y}^{-1}\left(F_{X}\left(x_{n}\right)\right) \triangleq g\left(x_{n}\right),
$$

where $F_{Y}^{-1}(\cdot)$ is the inverse of $F_{Y}(\cdot)$ and $F_{X}(\cdot)$ is the standard Gaussian CDF. And in order to attain the target $\rho_{Y}(\cdot), \rho_{X}(\cdot)$ is chosen to be the solution of [7]

$$
\rho_{Y}(m)=\sum_{k=1}^{\infty} d_{k}^{2} \rho_{X}(m)^{k}
$$

where

$$
d_{k} \triangleq \int_{-\infty}^{\infty} g(x) H_{k}(x) f_{X}(x) d x
$$

$H_{k}(\cdot)$ is the $k$ th Hermite polynomial, and $f_{X}(\cdot)$ is the wellknown standard Gaussian probability density function (PDF). As shown in [7], the coefficients $\left\{d_{k}^{2}\right\}_{k=1}^{\infty}$ decay rapidly. The number of coefficients required to solve (3) accurately depends on the probabilistic structure of $\left\{Y_{n}\right\}$.

Additional concerns have to be addressed. First, some pairs $\left\{F_{Y}(\cdot), \rho_{Y}(\cdot)\right\}$ are incompatible and can not be realized by any simulation scheme [7]. The realizable range of $\rho_{Y}(\cdot)$ depends on $F_{Y}(\cdot)$ and is given by [7]

$$
\frac{1}{\sigma^{2}}\left[\int_{0}^{1} F_{Y}^{-1}(u) F_{Y}^{-1}(1-u) d u-\mu^{2}\right] \leq \rho_{Y}(m) \leq 1 .
$$

Second, even when the target $\rho_{Y}(\cdot)$ satisfies (5), the corresponding $\rho_{X}(\cdot)$ obtained from (3) may not be legitimate (nonnegative definite) [7]. In this case, it is impossible to achieve the target $\rho_{Y}(\cdot)$ by using the scheme in Fig. 1. As an approximate solution, one has to find a legitimate $\rho_{X}(\cdot)$ so that the resulting $\rho_{Y}(\cdot)$ will be close in some sense to its target value. Different approaches can be used to reduce the error between the target and achieved values of $\rho_{Y}(\cdot)$. Refer to [7] for a detailed explanation on some of them.

The main concern with the standard method is that it requires $F_{Y}^{-1}(\cdot)$, both to find $\rho_{X}(\cdot)$ via (3) and to apply the nonlinearity (2) that produces $\left\{y_{n}\right\}$. The problem is that $F_{Y}^{-1}(\cdot)$ is sometimes not available. For instance, the gamma and beta CDFs, which find large applicability in engineering, have no closed-form inverse [8]. In such cases, the use of the standard method requires the numerical inversion of the target $\mathrm{CDF}$, rendering the method more intricate and less accurate. Next, we overcome these difficulties by proposing a novel method in which the knowledge of $F_{Y}^{-1}(\cdot)$ is dispensable.

\section{NOVEL METHOD}

For clarity, we start with a description of the novel method, postponing its theoretical background until the next section. The proposed method is as follows:

1) Generate an $L$-sample realization sequence $\left\{x_{n}\right\}$ for a zero-mean unit-variance Gaussian process $\left\{X_{n}\right\}$ with an appropriate ACC $\rho_{X}(\cdot)$.

2) Generate an $L$-sample realization sequence $\left\{z_{n}\right\}$ for an i.i.d. process $\left\{Z_{n}\right\}$ having $\operatorname{CDF} F_{Y}(\cdot)$.

3) Rearrange the samples in $\left\{z_{n}\right\}$ so as to match the rank of the samples in $\left\{x_{n}\right\}$, forming $\left\{y_{n}\right\}$.
The scheme is illustrated in Fig. 2 and detailed in the next paragraphs.

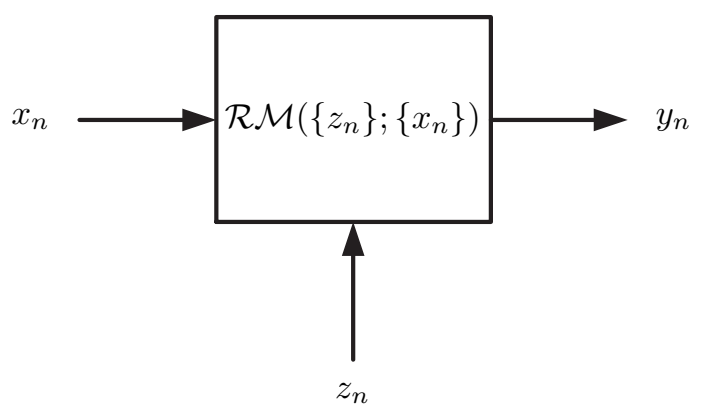

Fig. 2. The novel method for generating autocorrelated non-Gaussian sequences.

In step $1, \rho_{X}(\cdot)$ is chosen in the same way of the standard method, i.e., as being the solution of (3). Note that solving (3) depends on $d_{k}$, given in (4), which depends ultimately on $F_{Y}^{-1}(\cdot)$-recall that $g(\cdot) \triangleq F_{Y}^{-1}\left(F_{X}(\cdot)\right)$. Of course, one may use (4) if $F_{Y}^{-1}(\cdot)$ is available. On the other hand, by making in (4) the substitution of variables

$$
x=g^{-1}(y)=F_{X}^{-1}\left(F_{Y}(y)\right) \triangleq h(y),
$$

and after some algebraic manipulations, we obtain an alternative expression for $d_{k}$ that does not require $F_{Y}^{-1}(\cdot)$, namely

$$
d_{k}=\int_{S_{Y}} y H_{k}(h(y)) f_{X}(h(y)) \dot{h}(y) d y,
$$

where $\dot{h}(\cdot)$ denotes the first derivative of $h(\cdot)$ and $S_{Y}$ is the support of the PDF of $Y_{n}$. Since the inverse of the standard Gaussian CDF is given by

$$
F_{X}^{-1}(u)=\sqrt{2} \operatorname{erf}^{-1}(-1+2 u)
$$

where $\operatorname{erf}^{-1}(\cdot)$ is the inverse error function, it follows from (6) that

$$
h(y)=\sqrt{2} \operatorname{erf}^{-1}\left(-1+2 F_{Y}(y)\right) .
$$

In addition, it is easy to show that

$$
\dot{h}(y)=\sqrt{2 \pi} \exp \left[\operatorname{erf}^{-1}\left(-1+2 F_{Y}(y)\right)^{2}\right] f_{Y}(y),
$$

where $f_{Y}(\cdot)$ is the PDF of $Y_{n}$.

As in the standard method, $\rho_{Y}(\cdot)$ must satisfy (5) as a necessary condition for the resulting $\rho_{X}(\cdot)$ to be legitimate. But this condition is again not sufficient. Should $\rho_{X}(\cdot)$ not be legitimate, the same techniques used in the standard method to find a legitimate $\rho_{X}(\cdot)$ that minimizes the error in $\rho_{Y}(\cdot)$ can be used in the proposed method as well. Refer to [7] for some of those techniques.

Step 2 consists in drawing $L$ independent samples from $F_{Y}(\cdot)$. This can be accomplished by any method of random number generation, such as the percentile transformation method or the rejection method [9]. The former requires $F_{Y}^{-1}(\cdot)$, the latter does not. Thus, should $F_{Y}^{-1}(\cdot)$ be known, one can use either methods. Otherwise, one can use the rejection method. It is noteworthy that standard computing softwares include random number generators for most distri- 
butions in engineering.

Step 3 is a block-wise operation. We call it rank matching, since it takes two sequences with identical lengths, in our case $\left\{z_{n}\right\}$ and $\left\{x_{n}\right\}$, and rearrange the samples in $\left\{z_{n}\right\}$ to form a new sequence $\left\{y_{n}\right\}$, so that the samples in $\left\{y_{n}\right\}$ exactly match the rank of the samples in $\left\{x_{n}\right\}$, that is, their minima occur in the same position, their second minima occur in the same position, and so on. We say that $\left\{y_{n}\right\}$ is rank-matched to $\left\{x_{n}\right\}$. Correspondingly, we introduce the notation

$$
\left\{y_{n}\right\}=\mathcal{R} \mathcal{M}\left(\left\{z_{n}\right\} ;\left\{x_{n}\right\}\right) .
$$

The rank matching operation can be easily implemented in standard computing softwares by means of built-in ordering routines. In Matlab, for instance, a possible implementation is

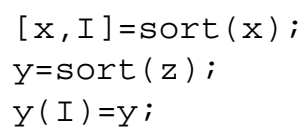

\section{THEORETICAL BACKGROUND}

In the proposed method, since the output sequence is a mere rearrangement of $L$ samples drawn i.i.d. from $F_{Y}(\cdot)$ (steps 2 and 3), then it certainly complies with the target CDF. However, it is not equally clear why the rearrangement proposed in step 3 should also comply with the target ACC. In this section, we answer this question by showing that the proposed rearrangement cause the output process of the novel method to converge in probability to that of the standard method as the sequence length $L$ goes to infinity.

To begin with, recall that the standard and novel methods start both with the same input Gaussian process $\left\{X_{n}\right\}, 1 \leq$ $n \leq L$. In order to distinguish the resulting output processes, we shall enhance the notation slightly, denoting the output process of the standard method by $\left\{\hat{Y}_{n}\right\}$, and that of the novel method by $\left\{\check{Y}_{n}\right\}, 1 \leq n \leq L$. The novel method still includes an additional process $\left\{Z_{n}\right\}, 1 \leq n \leq L$.

From (2), each variate $\hat{Y}_{n}$ is given by $\hat{Y}_{n}=g\left(X_{n}\right)$. Since $g(\cdot)$ is monotone nondecreasing [7], $\left\{\hat{Y}_{n}\right\}$ happens to be rankmatched to $\left\{X_{n}\right\}$, i.e., their minima occur in the same position, their second minima occur in the same position, and so on. In the same way, from (11), $\left\{\check{Y}_{n}\right\}$ is given by $\left\{\check{Y}_{n}\right\}=$ $\mathcal{R} \mathcal{M}\left(\left\{Z_{n}\right\} ;\left\{X_{n}\right\}\right)$, which is clearly also rank-matched to $\left\{X_{n}\right\}$. Thus the processes $\left\{\hat{Y}_{n}\right\}$ and $\left\{\check{Y}_{n}\right\}$ are rank-matched to each other.

Of course, rank-matched processes may be quite different in general. However, if these processes have additionally the same order statistics, then they are indeed identical (identical sample orders + identical sample sets $=$ identical sequences). We are not suggesting that $\left\{\hat{Y}_{n}\right\}$ and $\left\{\check{Y}_{n}\right\}$ have the same order statistics - they do not. But their order statistics converge in probability as $L$ goes to infinity, as shown next.

First, let us introduce some notation. As mentioned before, each of the processes $\left\{\hat{Y}_{n}\right\},\left\{\check{Y}_{n}\right\}$, and $\left\{Z_{n}\right\}$ is a set of $L$ variates. Now, define $\hat{Y}_{(1)}$ as the minimum of $\left\{\hat{Y}_{n}\right\}, \hat{Y}_{(2)}$ as the second minimum of $\left\{\hat{Y}_{n}\right\}$, and so on. In the same way, define $\check{Y}_{(1)}$ as the minimum of $\left\{\check{Y}_{n}\right\}, Z_{(1)}$ as the minimum of $\left\{Z_{n}\right\}, \check{Y}_{(2)}$ as the second minimum of $\left\{\check{Y}_{n}\right\}, Z_{(2)}$ as the second minimum of $\left\{Z_{n}\right\}$, and so on. The new sets of variates
$\left\{\hat{Y}_{(n)}\right\},\left\{\check{Y}_{(n)}\right\}$, and $\left\{Z_{(n)}\right\}$ are called the order statistics of $\left\{\hat{Y}_{n}\right\},\left\{\check{Y}_{n}\right\}$, and $\left\{Z_{n}\right\}$, respectively. Note that $\hat{Y}_{(1)} \leq \hat{Y}_{(2)} \leq$ $\cdots \leq \hat{Y}_{(L)}, \check{Y}_{(1)} \leq \check{Y}_{(2)} \leq \cdots \leq \check{Y}_{(L)}$, and $Z_{(1)} \leq Z_{(2)} \leq$ $\cdots \leq Z_{(L)}$.

The aim is to compare $\left\{\hat{Y}_{(n)}\right\}$ with $\left\{\check{Y}_{(n)}\right\}$. From $\left\{\check{Y}_{n}\right\}=$ $\mathcal{R} \mathcal{M}\left(\left\{Z_{n}\right\} ;\left\{X_{n}\right\}\right)$, it is clear that $\left\{\check{Y}_{(n)}\right\}=\left\{Z_{(n)}\right\}$, since $\left\{\check{Y}_{n}\right\}$ is a rearrangement of $\left\{Z_{n}\right\}$. Thus, it suffices to compare $\left\{\hat{Y}_{(n)}\right\}$ with $\left\{Z_{(n)}\right\}$. By construction, the variates in $\left\{Z_{n}\right\}$ are independent. In contrast, the variates in $\left\{\hat{Y}_{n}\right\}$ are somehow correlated, since they result from a memoryless nonlinearity applied to an autocorrelated Gaussian process. However, all of the variates in both $\left\{Z_{(n)}\right\}$ and $\left\{\hat{Y}_{(n)}\right\}$ follow the same target CDF $F_{Y}(\cdot)$. From the asymptotic theory of order statistics, it is known that the $n$th order statistic, $1 \leq n \leq L$, of a set of $L$ variates following a given $\mathrm{CDF}$, say $F_{Y}(\cdot)$, converges in probability to the quantile $F_{Y}^{-1}\left(\lim _{L \rightarrow \infty} \frac{n}{L}\right)$ as $L$ goes to infinity, regardless of the amount of correlation between the variates ${ }^{3}$ [10]. Thus, it follows that both $Z_{(n)}$ and $\hat{Y}_{(n)}$, $1 \leq n \leq L$, converge in probability to the same value $F_{Y}^{-1}\left(\lim _{L \rightarrow \infty} \frac{n}{L}\right)$ as $L$ goes to infinity.

We have shown that the output processes of the standard and novel methods have not only the same rank statistics but also order statistics that converge in probability to each other as $L$ goes to infinity. The greater the $L$, the closer in probability the output processes, and thus the better the expected performance of the novel method. Of course, it remains to know whether the convergence is fast enough to render the novel method accurate for values of $L$ of practical interest, e.g., $L=2^{20}$. Next, we answer this question affirmatively by means of extensive examples.

A last remark is required. By assumption, both the standard method and our method start with the generation of a stationary, ergodic, autocorrelated Gaussian sequence. In the standard method, a memoryless nonlinear transformation is then applied to the Gaussian sequence to produce the output non-Gaussian sequence. Therefore, the output sequence is also stationary and ergodic. On the other hand, in our method, it seems to be difficult to assess these issues in an exact manner. However, as shown here, the output sequence of our method converges in probability to that of the standard method as the sequence size goes to infinity. In this sense, our method provides sequences that are asymptotically stationary and asymptotically ergodic. The larger the sequence size, the higher the degree of stationarity and ergodicity.

\section{EXAMPLES}

We shall consider five CDFs (triangle, Rayleigh, Laplace, uniform, and exponential) and four ACCs (linearly decaying, exponentially decaying, exponentially-decaying cosine, and Bessel $^{4}$ ). We have intentionally selected only CDFs with known inverse, for the aim is to compare the performance of the novel method with the best-case performance of the standard method, when the latter is not subject to inaccuracies due to the numerical inversion of the target CDF. Of course,

\footnotetext{
${ }^{3}$ The index $n$ is allowed to be a function of $L$, so that $\lim _{L \rightarrow \infty} \frac{n}{L}$ may range from 0 to 1 .

${ }^{4}$ We have used the Bessel function of the first kind and zeroth order, $J_{0}(\cdot)$.
} 
should the inverse be unknown, the standard method loses accuracy, whereas the novel method is not affected.

Table I shows the CDFs, the inverse CDFs, and the coefficients $\left\{d_{k}\right\}_{k=1}^{7}$ obtained via (4) or (7). We have dropped the coefficients beyond the seventh, which are indeed negligible, and normalized $\left\{d_{k}\right\}_{k=1}^{7}$ to ensure that $\rho_{Y}(0)=\sum_{k=1}^{7} d_{k}^{2}=$ 1. The same procedure has been used in [7]. Indeed, the coefficients in Table I coincide with those in [7, Table I].

In each of the cases, the coefficients $\left\{d_{k}\right\}_{k=1}^{7}$ have been replaced into (3) to compute the appropriate $\rho_{X}(\cdot)$ that leads to the target $\rho_{Y}(\cdot)$, and an autocorrelated Gaussian sequence with $L=2^{20}$ samples has been generated accordingly, using the autoregressive method. The simulations have been run in Matlab. In those cases where $\rho_{X}(\cdot)$ happened not to be legitimate (nonnegative definite), the spectrum truncation method has been applied to render it legitimate. Refer to [7] for explanations on the spectrum truncation method.

The Gaussian sequences have been then used to produce the output sequences according to the standard and novel methods, as explained in Sections II and III. Table II lists the distortion

$$
\delta \triangleq \sum_{m} w_{m}\left(\rho_{Y}^{T}(m)-\rho_{Y}^{A}(m)\right)^{2}
$$

observed in each case between the achieved ${ }^{5}\left(\rho_{Y}^{A}(\cdot)\right)$ and target $\left(\rho_{Y}^{T}(\cdot)\right)$ values of $\rho_{Y}(\cdot)$, where

$$
w_{m}=1-\frac{\min (|m|, 100)}{100}
$$

is a triangle weighting sequence. The above distortion measure and a similar weighting sequence have been used in [7]. Note in all of the cases that the distortions of the two methods are very small and, more important, very similar to each other, which confirms the theory behind the novel method. As in [7], the largest distortion appear in the exponential case. This is expected, because the distortion should increase as $d_{1}^{2}$ decreases [7] and the exponential CDF presents the smallest $d_{1}^{2}$, as shown in Table I.

The achieved and target values of $\rho_{Y}(\cdot)$ are plotted in Fig. 3 for the triangle $\mathrm{CDF}$ and in Fig. 4 for the exponential $\mathrm{CDF}^{6}$. The target $\rho_{Y}(\cdot)$ is shown in solid lines, and the achieved $\rho_{Y}(\cdot)$ is shown in dashed lines for the novel method and in dotted lines for the standard method. Note again that the performances of the standard and novel methods are excellent and indistinguishable, and slightly poorer in the exponential case (Fig. 4).

In order to assess the rate of convergence between the output sequences of the standard and novel methods, we have run both methods for different sequence lengths, namely $L=2^{10}, 2^{11}, 2^{12}, \ldots, 2^{20}$. From the resulting output sequences, we have then estimated the average relative deviation between the samples generated by the methods. All of the samples have been considered in the estimation. The results are shown in Fig. 5. Note in all of the cases how the deviation

\footnotetext{
${ }^{5}$ The achieved value of $\rho_{Y}(\cdot)$ has been estimated by sample averaging the simulated output sequences.

${ }^{6}$ The corresponding curves for the Rayleigh, Laplace, and uniform CDFs were observed to be quite similar to those for the triangle CDF and have been omitted for the lack of space.
}

between the output samples of the two methods decreases steadily as the sequence length increases. Also note that this deviation does not exceed $3 \%$ for $L=2^{20}$.

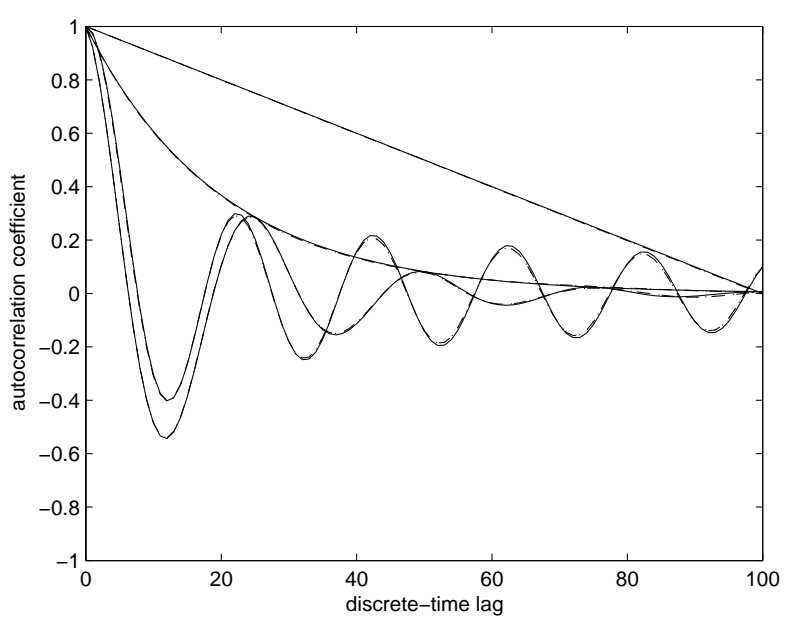

Fig. 3. Autocorrelation coefficient for triangle distribution (target: solid; standard method: dot; novel method: dash).

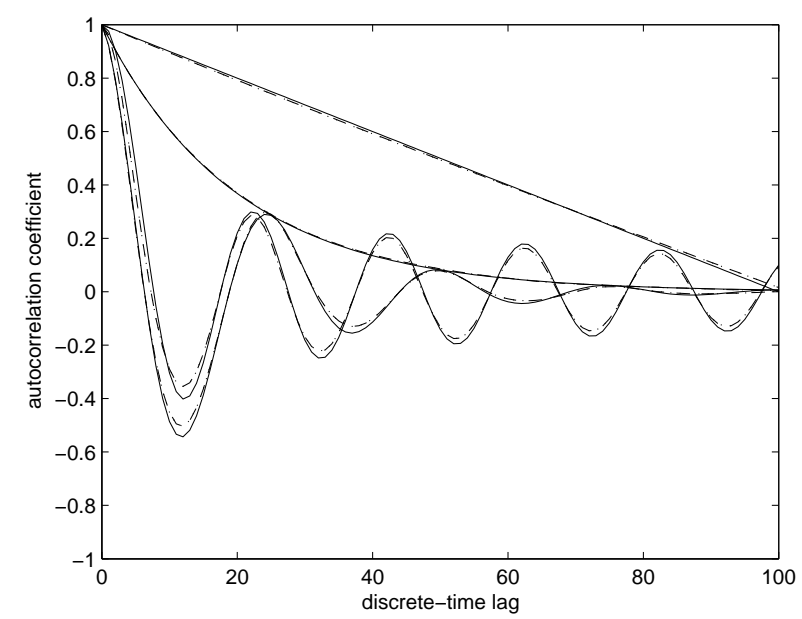

Fig. 4. Autocorrelation coefficient for exponential distribution (target: solid; standard method: dot; novel method: dash).

\section{CONCLUSIONS}

We proposed a novel method for generating random sequences matching a target non-Gaussian distribution and a target autocorrelation. In the proposed scheme, samples matching the target distribution are drawn independently and then rearranged to match the target autocorrelation. The main advantage of our method is that it dispenses with the inversion of the target distribution, thus easily accommodating the distributions with unknown inverse. In contrast, the standard method requires the inversion by construction and has to resort to numerical approximations when the inverse is unknown in closed form. We have compared our method to the standard method in its best-case performance (known inverse), and indistinguishable 


\begin{tabular}{|c|c|c|c|c|c|}
\hline & Triangle & Rayleigh & Laplace & Uniform & Exponential \\
\hline$f_{Y}(y)$ & $1-|y|,|y| \leq 1$ & $y e^{-y^{2} / 2}, y \geq 0$ & $\frac{1}{2} e^{-|y|},|y|<\infty$ & $1,0 \leq y \leq 1$ & $e^{-y}, y \geq 0$ \\
\hline$F_{Y}(y)$ & $\begin{array}{lc}\frac{1}{2}\left(1+2 y-y^{2}\right) & 0<y \leq 1 \\
\frac{1}{2}\left(1+2 y+y^{2}\right) & -1 \leq y \leq 0\end{array}$ & $1-e^{-y^{2}}$ & $\left\{\begin{array}{cl}\frac{e^{x}}{2} & x<0 \\
\frac{e^{-x}}{2}\left(-1+2 e^{x}\right) & x \geq 0\end{array}\right.$ & $y$ & $1-e^{-x}$ \\
\hline$F_{Y}^{-1}(y)$ & $\begin{array}{cl}-1+\sqrt{2 y} & 0<y \leq 1 / 2 \\
1-\sqrt{2(1-y)} & 1 / 2<y \leq 1 \\
\end{array}$ & $\sqrt{-\ln (1-y)}$ & $\begin{array}{cl}\ln (2 y) & 0<y \leq 1 / 2 \\
-\ln (2(1-y)) & 1 / 2<y \leq 1\end{array}$ & $y$ & $-\ln (1-y)$ \\
\hline$d_{1}^{2}$ & 0.9927701 & 0.9724690 & 0.9632098 & 0.9550627 & 0.8157660 \\
\hline$d_{2}^{2}$ & 0.0 & 0.0264400 & 0.0 & 0.0 & 0.1773910 \\
\hline$d_{3}^{2}$ & 0.0071243 & 0.0010467 & 0.0352063 & 0.0044768 & 0.0066847 \\
\hline$d_{4}^{2}$ & 0.0 & 0.0000113 & 0.0 & 0.0 & 0.0001343 \\
\hline$d_{5}^{2}$ & 0.0000010 & 0.0010467 & 0.0013255 & 0.0044768 & 0.0000169 \\
\hline$d_{6}^{2}$ & 0.0 & 0.0000017 & 0.0 & 0.0 & 0.0000073 \\
\hline$d_{7}^{2}$ & 0.0000100 & 0.0000007 & 0.0002592 & 0.0006662 & 0.0 \\
\hline
\end{tabular}

TABELA I

CDFs, INVERSE CDFS, AND COEFFICIENTS $d_{k}^{2}$.

\begin{tabular}{|c|c|c|c|c|c|c|c|c|c|c|}
\hline \multirow[t]{2}{*}{$\rho_{Y}(m)$} & \multicolumn{2}{|c|}{ Triangle } & \multicolumn{2}{|c|}{ Rayleigh } & \multicolumn{2}{|c|}{ Laplace } & \multicolumn{2}{|c|}{ Uniform } & \multicolumn{2}{|c|}{ Exponential } \\
\hline & standard & novel & standard & novel & standard & novel & standard & novel & standard & novel \\
\hline$\overline{1-\min (|m|, 100) / 100}$ & $3.82 \mathrm{e}-5$ & $3.82 \mathrm{e}-5$ & $4.51 \mathrm{e}-4$ & $4.89 \mathrm{e}-4$ & $2.70 \mathrm{e}-4$ & $3.80 \mathrm{e}-4$ & $7.55 \mathrm{e}-4$ & $\overline{7.72 \mathrm{e}-4}$ & $2.32 \mathrm{e}-3$ & $1.94 \mathrm{e}-3$ \\
\hline $\exp (-0.05|m|)$ & $2.90 \mathrm{e}-4$ & $2.95 \mathrm{e}-4$ & $2.02 \mathrm{e}-4$ & $2.05 \mathrm{e}-4$ & $2.92 \mathrm{e}-4$ & $2.76 \mathrm{e}-4$ & $8.94 \mathrm{e}-5$ & $8.89 \mathrm{e}-5$ & $2.78 \mathrm{e}-4$ & $2.41 \mathrm{e}-4$ \\
\hline $\exp (-0.05|m|) \cos (0.25|m|)$ & $3.41 \mathrm{e}-4$ & $3.43 \mathrm{e}-4$ & $1.49 \mathrm{e}-4$ & $1.51 \mathrm{e}-4$ & $9.14 \mathrm{e}-4$ & $9.63 \mathrm{e}-4$ & $2.84 \mathrm{e}-4$ & $2.79 \mathrm{e}-4$ & $1.13 \mathrm{e}-2$ & $1.12 \mathrm{e}-2$ \\
\hline$J_{0}(0.1 \pi|m|)$ & $2.91 \mathrm{e}-3$ & $2.92 \mathrm{e}-3$ & $3.60 \mathrm{e}-3$ & $3.62 \mathrm{e}-3$ & $6.33 \mathrm{e}-3$ & $6.23 \mathrm{e}-3$ & $6.54 \mathrm{e}-3$ & $6.56 \mathrm{e}-3$ & $5.60 \mathrm{e}-2$ & $5.60 \mathrm{e}-2$ \\
\hline
\end{tabular}

TABELA II

DISTORTION $\delta$ WITH TRIANGLE WEIGHTING AND SPECTRUM TRUNCATION.

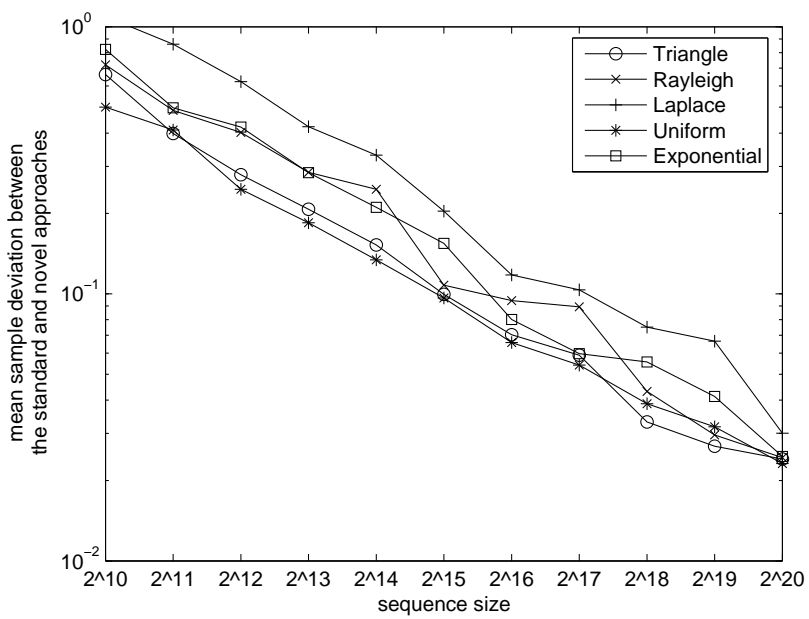

Fig. 5. Mean relative deviation between output samples of standard and novel methods, as a function of sequence size.

results have been observed in all of the cases. Besides communications, our method finds applicability in many different areas concerned with non-Gaussian processes.

\section{REFERENCES}

[1] M. J. Levin, "Generation of sampled gaussian time series having a specified correlation function," IRE Trans. Inform. Theory, vol. IT-6, pp. 545-548, Dec. 1960.

[2] T. G. Newman and P. L. Odell, The Generation of Random Variates. London: Charles Griffin, 1971.

[3] Y. Chen and R. Blum, "Efficient algorithms for sequence detection in non-Gaussian noise with intersymbol interference," IEEE Trans. Commun., vol. 48, no. 8, pp. 1249-1252, Aug. 2000.
[4] H. Poor and M. Tanda, "Multiuser detection in flat fading non-Gaussian channels," IEEE Trans. Commun., vol. 50, no. 11, pp. 1769-1777, Nov. 2002.

[5] C. Luschi and B. Mulgrew, "Nonparametric trellis equalization in the presence of non-Gaussian interference," IEEE Trans. Commun., vol. 51, no. 2, pp. 229-239, Feb. 2003.

[6] U. G. Gujar and R. J. Kavanagh, "Generation of random signals with specified probability density functions and power density spectra," IEEE Trans. Automat. Contr., vol. AC-13, pp. 716-719, Dec. 1968.

[7] B. Liu and D. C. Munson, "Generation of a random sequence having a jointly specified marginal distribution and autocovariance," IEEE Trans. Acoust., Speech, Signal Process., vol. ASSP-30, no. 6, pp. 973-983, Dec. 1982.

[8] K. V. Bury, Statistical Distributions in Engineering. Cambridge: Cambridge University Press, 1999.

[9] A. Papoulis and S. U. Pillai, Probability, Random Variables and Stochastic Processes, 4th ed. New York: McGraw-Hill, 2002.

[10] H. A. David and H. N. Nagaraja, Order Statistics, 3rd ed. WileyInterscience, 2003. 